\title{
\begin{tabular}{l|l} 
POLITIQUES \& & Politiques et management public
\end{tabular}
}

\section{La gouvernance des fédérations d'associations chargées d'une mission de service public : le cas des fédérations sportives françaises}

Governance of non profit governing bodies in charge of public mission service. The case of french sport governing bodies

\section{Emmanuel Bayle}

\section{OpenEdition \\ Journals}

Édition électronique

URL : http://journals.openedition.org/pmp/1992

DOI : $10.4000 /$ pmp. 1992

ISSN : 2119-4831

\section{Éditeur}

Institut de Management Public (IDPM)

\section{Édition imprimée}

Date de publication : 15 janvier 2010

Pagination : 121-142

ISSN : 0758-1726

Référence électronique

Emmanuel Bayle, «La gouvernance des fédérations d'associations chargées d'une mission de service public : le cas des fédérations sportives françaises », Politiques et management public [En ligne], Vol. 27/1 | 2010, mis en ligne le 15 janvier 2012, consulté le 30 avril 2019. URL : http:// journals.openedition.org/pmp/1992 ; DOI : 10.4000/pmp.1992 


\title{
LA GOUVERNANCE DES FÉDÉRATIONS D'ASSOCIATIONS CHARGÉES D'UNE MISSION DE SERVICE PUBLIC : LE CAS DES FÉDÉRATIONS SPORTIVES FRANÇAISES
}

\author{
Emmanuel BAYLE ${ }^{1}$
}

Résumé

Mots clés

Summary

\begin{abstract}
La notion de gouvernance des organisations recouvre " l'ensemble des mécanismes organisationnels qui ont pour effet de délimiter les pouvoirs et d'influencer les décisions des dirigeants, autrement dit qui gouvernent leur conduite et définissent leur espace discrétionnaire »(Charreaux, 1997). Nous distinguons avec Henry (2005) trois dimensions : la gouvernance organisationnelle, politique et systémique. L'article est centré sur la gouvernance organisationnelle en interaction avec les deux autres formes de gouvernance. II s'intéresse essentiellement à la question de l'efficacité de la gouvernance en lien avec le rôle des principaux dirigeants des fédérations sportives françaises (association en charge d'une mission de service public : promouvoir, organiser et développer leur-s- discipline-ssportive-s-) au regard des influences auxquelles ils doivent faire face. Depuis dix ans sous l'influence de la doctrine Carver (1997, 2001), les agences ministérielles en charge du sport en Grande-Bretagne, en Australie, en Nouvelle-Zélande et surtout au Canada ont identifié l'amélioration de la gouvernance comme une priorité stratégique pour les (grandes) associations. De nouvelles pratiques ont vu le jour en la matière transformant radicalement les pratiques traditionnelles originelles. Nous analyserons leurs intérêts pour les fédérations sportives françaises qui connaissent depuis de nombreuses années un certain nombre de difficultés en la matière : une politique et des pratiques de gouvernance mal définies, des professionnels qui peuvent prendre, officiellement ou officieusement, la direction politique de ces organisations ; des présidents parfois omnipotents sans l'existence de CA exerçant de réels contrepouvoirs ; une absence de clarté dans la délégation managériale ; un risque de rupture, de sclérose et de conflits entre le sommet (le siège fédéral) et la base (les clubs - mandants) ; un rôle incitatif et de contrôle ambivalent du ministère de tutelle.
\end{abstract}

Fédérations d'associations, Gouvernance, Mission de service public, pouvoir, tutelle publique.

\section{Governance of non profit governing bodies in charge of public mission service. The case of french sport governing bodies}

The concept of governance of organisations can be define as " all organisational's mechanisms which have the effect of delimitate power and influence decisions of managers, that is govern their behaviour and the span of their capacities" (Chareraux, 1997). We distinguish, with Henry (2005), three dimensions of this concept : organisational, political and systemic governance. This article is centred on organisational governance in link with the two other forms of governance. It deals with effectiveness of governance in relation with the role of managers

\footnotetext{
${ }^{1}$ Professeur à l'Institut d'Administration des Entreprises de l'Université Jean Moulin Lyon 3 Centre de recherche en gestion Magellan (EA 3713)
}

Revue POLITIQUES ET MANAGEMENT PUBLIC, Volume 27, n 1, 2010. C Institut de Management Public - 2010. 
(elected and paid) of French sport federations (in charge of a public mission service: promote, organise and develop their sport). Since ten years under the influence of Carver's publications $(1997,2001)$, ministries in charge of sport in Great Britain, in Australia, in New Zealand and above all in Canada have identified the improvement of governance as strategic priority of big voluntary organisations. In these countries, new practices of governance have emerged which have transformed traditional and former governance practices. We analysed their interests of French sport federations which faced some difficulties in this field : a policy and governance's practices are not well defined, professional managers who can take officially or not the political leadership of these organisations ; president who concentrate all the powers without counter powers of board of administration in front of them ; a managerial's delegation not clear ; a risk of beach of contact and conflicts between headquarters of the federations and clubs (local associations) ; a role of control of ministry of sport which is not very clear and effective.

Key words Governance, Non profit governing bodies, Public mission service, Power, State delegation. 
Introduction
Si la gouvernance des (grandes) entreprises et celle des organisations politiques sont régulièrement mises en cause par le marché, les médias, l'opinion publique, celle des associations est moins connue voire plus secrète. Cependant, cette situation tend à évoluer en raison du poids social, économique et politique grandissant que représente le secteur associatif français (Demoustier, 2001 ; Lipietz, 2001). Souvent coproducteur de politiques publiques et partenaires d'entreprises (sponsors et mécènes), les associations et notamment les plus grandes d'entre elles, voient leur gouvernance et leurs performances (économiques, sociales et sociétales) plus régulièrement interrogées voire contestées que ce soient pour des questions de vie démocratique réelle, de recherche de lucrativité excessive voire parfois de malversations (Kaltenbach, 1996 ; rapports annuels de la Cour des comptes). Depuis, ces quinze dernières années, la montée en puissance du concept polysémique de gouvernance est significative à la fois dans la terminologie managériale des responsables associatifs mais aussi à travers la parution de nombreux travaux d'études spécifiques (par exemple en France rapport Ernst et Young, 2006 ; ouvrage de l'Institut Français des Administrateurs, 2009) et de recherches notamment anglosaxonnes (voir bibliographie).

Chaque sous secteur associatif a souvent ses caractéristiques et ses problématiques propres de gouvernance (santé, social, culture, sport ...) qui s'explique par son histoire, le poids de la tutelle publique, par les formes de professionnalisation choisies et les pressions (sociales, économiques, institutionnelles, médiatiques...) qui s'y exercent (Queinnec et Igallens, 2004). Malgré la grande hétérogénéité du secteur associatif, les problèmes de gouvernance des associations s'inscrivent dans un terreau commun (absence de but lucratif, gestion démocratique, dyarchie élus-bénévoles et salariés, complexité des mécanismes incitatifs). La gouvernance pour les grandes associations est un sujet managérial central à la fois en tant que facteur clé de performance (Bayle, 2007) mais aussi comme une des dimensions permettant caractériser un comportement socialement responsable.

Cet article vise à étudier, à partir d'une approche qualitative et quantitative, les modalités, les problèmes et perspectives d'évolution de la gouvernance des grandes fédérations d'associations dans un des secteurs importants du monde associatif. La question des modalités de décision, de pouvoir et de conflits d'intérêts, du contrôle en matière de gouvernance est particulièrement sensible afin de mieux comprendre qui décide, qui manage et qui contrôle dans un univers où sphères bénévole et professionnel cohabitent. Cette question complexe se pose en interne mais aussi dans la relation avec la tutelle. Elle doit être prise en compte dans une perspective dynamique et socio-historique afin de mieux comprendre les enjeux, les rationalités et les systèmes de légitimité qui s'y exercent. 
Nous définissons tout d'abord le concept de gouvernance et son application aux fédérations sportives. Puis, nous dressons un état des lieux des pratiques de gouvernance dans les fédérations sportives françaises (FSF). Les éléments de réponse donnés dans le monde anglosaxon aux problèmes de gouvernance des associations sont, ensuite, étudiés. Finalement, leur intérêt pour les FSF est débattu.

\section{Le concept de gouvernance et son application aux fédérations sportives}

Avec Henry (2005), nous définissons la gouvernance autour de trois dimensions (1) : la gouvernance organisationnelle, politique et systémique. Nous avons choisi de focaliser notre recherche sur la gouvernance organisationnelle en interaction avec les deux autres formes de formes de gouvernance. Nous expliquons la méthodologie adoptée pour l'étude de la gouvernance des FSF (2).

\section{Les trois dimensions du concept}

La notion de gouvernance des organisations recouvre " l'ensemble des mécanismes organisationnels qui ont pour effet de délimiter les pouvoirs et d'influencer les décisions des dirigeants, autrement dit qui gouvernent leur conduite et définissent leur espace discrétionnaire " (Charreaux, 1996). Au-delà de cette définition, nous distinguons, avec Henry (2005), trois dimensions du concept de gouvernance : la gouvernance : organisationnelle, politique et systémique.

La première (organisationnelle) concerne l'exercice du pouvoir au sein des organisations, les processus de décision et la conduite de la politique (structures, processus et contrôle) en vue d'améliorer la performance d'une organisation et l'atteinte de ses objectifs. Ainsi, il existe une proximité avec l'approche en termes de corporate governance (Charreaux, 1997, 2002 ; Gomez, 1996, 2001 ; Perez, 2003 ; Finet, 2005, Pesqueux, 2007). Cependant, cette grille de lecture s'avère inadaptée à la compréhension du fonctionnement des associations à but non lucratif. En effet, d'une part, la nature multidimensionnelle des objectifs et de la performance de ces organisations est non réductible à la seule dimension financière. D'autre part, la multiplicité des acteurs engagés dans l'organisation et la gestion du sport (clubs, fédérations nationales et internationales, ministère des sports...) complexifie les processus de prise de décision et d'exercice du pouvoir. Enfin, l'ambiguïté de la relation entre dirigeants élus (le plus souvent bénévoles) et cadres permanents rémunérés ou/et, parfois, mis à disposition par l'Etat rend problématique la répartition des rôles (politique/managérial/opérationnel) et du pouvoir de décision. Ces trois caractéristiques fondent la spécificité de la gouvernance des organisations à but non lucratif sur laquelle un certain nombre d'auteurs se sont déjà penchés notamment à travers une lecture via la théorie de l'agence (Fama \& Jensen 1983 ; Courtois, 1997) ${ }^{2}$.

La deuxième dimension (politique) concerne le rapport que les organisations associatives entretiennent vis-à-vis du champ politique au

\footnotetext{
${ }^{2}$ Dans le secteur sportif associatif, les travaux sur ce thème sont plus récents et très épars (Papadimitriou, 1999 ; Bayle, 2001). Cf. cependant l'ouvrage de synthèse de Hoye \& Cuskelly, 2006.
} 
double sens du terme. D'une part, il s'agit de rendre compte de la façon dont les institutions politiques (Etats, Union Européenne...) cherchent à influencer, voire à piloter le système sportif associatif via la mobilisation de dispositifs réglementaires, financiers et moraux. D'autre part, il s'agit d'analyser la question du pouvoir, non plus dans sa dimension intra, mais inter-organisationnelle. Des travaux, souvent sectoriels (culture, sanitaire et social, éducation...), en sociologie politique et en sciences politiques ont été menés en termes de gouvernance politique fondée sur l'étude des rapports entre État/Collectivités locales et associations souvent agent de mission de service public.

La troisième dimension (systémique) vise à rendre compte des situations d'interdépendance et des formes de régulation des relations interorganisationnelles et de la coordination des actions au sein d'un système complexe. L'idée de complexité renvoie à l'incertitude de l'environnement, à la multiplicité des parties prenantes présentant des intérêts souvent différents (public, associatif, marchand) et à la multiplicité des niveaux de coordination (du local au global). Sur le plan dynamique, la notion de gouvernance systémique marque le passage d'une régulation/coordination des actions centralisée, hiérarchique et verticale (gouvernement) à une régulation/coordination horizontale en termes de réseaux fondés sur le consensus/compromis et le pouvoir partagé (cf. dans le secteur du sport Bayle et Durand, 2004 ; Honta, 2007).

\section{Méthodologie d'étude la gouvernance organisationnelle des FSF}

L'article est centré sur la gouvernance organisationnelle des FSF en interaction avec les deux autres formes de gouvernance. C'est-à-dire que nous avons cherché à comprendre comment la gouvernance organisationnelle était influencée par les évolutions touchant la gouvernance politique mais aussi systémique. C'est pourquoi, la question des modes de changement et d'adaptation tenant à la commercialisation croissante et la managérialisation du secteur public est également au cœur de l'étude (Amis, Slack, \& Hinings, 2004; Kikulis, 2000; Kikulis, Slack, \& Hinings, 1992, 1995). Les pratiques de gouvernance représentent un des facteurs clés de la performance des fédérations d'associations (Deloitte et Touche, 2003 ; Bayle, 2007) en tant qu'élément majeur du leadership et de l'orientation stratégique de ces organisations (Adams et Perlmutter, 1995 ; Chait et al., 2004).

La gouvernance organisationnelle des fédérations d'associations peut se concevoir selon une double dimension, ce qui ajoute à la complexité du pilotage de ce type d'organisation : la gouvernance du siège (généralement parisien) et celle du réseau (du national au local), qui dépend lui-même d'un niveau international les fédérations internationales.

La première concerne principalement l'étude des relations entre le bureau (président, trésorier, principaux élus...), le conseil d'administration -CA(ou comité directeur -CD-et les principaux cadres salariés (Directeur-s-) et les cadres fonctionnaires mis à disposition par l'Etat (le Directeur 
Technique National). La plupart des travaux publiés en langue anglaise ${ }^{3}$ et très rarement en français (Mayaux, 1999) ont porté sur les types et rôles du CA (notamment Murray et al., 1992), sur l'efficacité du CA (Green, J.C. \& Griesinger, D. 1996 ; Papadimitriou, 1999 avec une application aux fédérations sportives grecques et l'ouvrage de synthèse de Hoye \& Cuskelly, 2006 concernant la gouvernance des organisations sportives) et sur l'équilibre idéal de pouvoir qu'il devrait y avoir entre le CA, le directeur et les cadres (Herman, R.D. \& Heimovics, R.D. 1991). Des travaux ont également montré, à travers l'étude des modalités d'exercice du pouvoir (cf. le concept de noyau stratégique de Mayaux, 1996) qui pouvaient se révéler très différents du fonctionnement statutaire. Bayle (1999) identifie ainsi quatre modes de gouvernements (Présidentiel fort ; Tandem ; Présidentiel divisé et Managérial) et a trouvé des modes de fonctionnement des CA assez similaires à ceux mis en évidence par Mayaux (1999) et Murray et al. (1992) à partir de travaux portant sur plusieurs secteurs d'activités associatifs. Mayaux identifie quatre types de CA : le "conseil dispositif de contrôle" dirige l'association et contrôle le travail des salariés.

Les administrateurs sont perçus comme des patrons jouant leur rôle d'employeurs et faisant parfois preuve d'autorité. Le "conseil outil" donne des conseils, des avis et des coups de main. Les administrateurs sont perçus par les salariés comme des collègues et des accompagnateurs. Le conseil d'administration est vu comme un lieu d'échange, de réflexion et de propositions, comme un groupe d'appui. Le "conseil façade" correspond à un système où le rôle du conseil d'administration est très limité et formel. Les réunions de conseil consistent souvent à entériner des décisions déjà prises par les salariés. Le "conseil militant" signifie que les administrateurs sont avant tout des militants convaincus et engagés. Ils se sentent garants de l'institution, de sa philosophie et de son éthique. Le conseil d'administration est perçu comme un organe politique".

La seconde dimension, peu étudiée par la littérature, fait plutôt référence à la coordination du réseau fédéral (siège national, structures régionales et départementales et associations/clubs locaux) qui a épousé le processus de décentralisation (ligues ou délégations régionales et comités départementaux étant les interlocuteurs des conseils régionaux et généraux et des services déconcentrés de l'Etat). Elle vise à définir comment mieux soutenir et accompagner les structures locales qui sont au contact direct des membres (licenciés) et plus largement à améliorer la qualité du maillage fédéral (et la qualité de la collaboration entre quatre niveaux d'intervention : national, régional, départemental et local).

Afin d'étudier les modalités, les problèmes et les évolutions possibles de la gouvernance organisationnelle des FSF - en charge d'une mission de service public-, la méthodologie qualitative et exploratoire s'appuie sur un suivi (juridique - notamment quant aux évolutions législatives en matière de gouvernance-, économique et sociologique) des problématiques de gouvernance du secteur d'activité (environ 100 fédérations d'associations

\footnotetext{
${ }^{3}$ Publiés principalement dans deux revues : Non profit management and leadership ; Non profit and voluntary sector quarterly.
} 
de tailles très différentes ${ }^{4}$ ) étudié depuis une quinzaine d'année (1993$2009^{5}$ ) et sur onze études de cas approfondies. Le suivi du secteur permet de comprendre les enjeux et les pratiques en termes de gouvernance organisationnelle mais aussi politique et systémique à travers un certain nombre de sources (revue de presse spécialisée, entretiens semi-directifs et ouverts avec les acteurs du secteur). Les onze études de cas ont été initiées pour six $^{6}$ d'entre elles durant la thèse de doctorat de gestion (Bayle, 1999) et pour cinq $^{7}$ d'entre elles dans le cadre d'une enquête qualitative réalisée pour le Comité national olympique et sportif français -CNOSF-, chargé de fédérer le mouvement sportif (2003) ; en 2005, une enquête menée dans le cadre d'une étude/ouvrage pour le CNOSF sur les pratiques de gouvernance et de management des fédérations, réalisée à partir d'un questionnaire auquel 60 fédérations sur 95 ont répondu, nous a permis de récolter des informations quantitatives.

Pour réaliser les cas, les données ont été recueillies par observations au siège des fédérations, entretiens avec les dirigeants et les cadres des FSF étudiées. Ces informations confrontées à la littérature nous ont permis de mieux comprendre le fonctionnement des CA (compositionrôle), des bureaux (profils socioprofessionnels des dirigeants et modalités d'implication et temps de travail) et des missions et des rôles (politique, managérial, opérationnel ${ }^{8}$ ) tenus par les principaux acteurs élus (président, trésorier, secrétaire général et vice-présidents) et salariés (directeurs et cadres). L'encadré 1 résume les principaux points analysés au niveau des enquêtes quantitative et qualitative.

\footnotetext{
${ }^{4}$ Le mouvement sportif associatif que chapeautent les fédérations représenterait environ 80000 emplois ETP. Au siège parisien, le total des comptes de résultats des plus grandes fédérations sont d'environ 140 millions d'euros pour la FF tennis ( 350 salariés) et 120 millions pour le football ( 200 salariés) ; les grandes fédérations olympiques (judo, gymnastique, athlétisme, basket-ball...) disposent de budget compris entre 10 et 20 millions d'euros et compte en moyenne une cinquantaine de salariés. Les fédérations olympiques de taille moyenne (badminton, escrime, taekwandoe, triathlon...) ont des budgets entre 4 et 8 millions d'euros (de 10 à une vingtaine de salariés. Les petites fédérations olympiques et les fédérations non olympiques moyennes et petites ont souvent des budgets inférieurs à 4 millions d'euros et moins de 10 salariés. Près de $50 \%$ des fédérations non olympiques soient plus de moitié de l'ensemble des fédérations ont entre 1 et 5 salariés (Bayle et Bruzek, 2005). Selon la même enquête, les fédérations olympiques consacrerait $16,5 \%$ de leur budget à la masse salariale et $17,5 \%$ pour les fédérations olympiques.

${ }^{5}$ L'auteur a été à un poste privilégié du sport français en travaillant au CNOSF de 1993 à 1994 et en qualité de consultant entre 1994-2000 dans un Centre spécialisé d'étude des fédérations (le Centre de droit et d'économie du sport).

${ }^{6}$ Les fédérations de basket-ball, de gymnastique, d'haltérophilie, de judo, de randonnée pédestre et de tennis disposant de tailles, de caractéristiques (olympique/non olympique; sport individuel/collectif) et de profils de performance différents.

7 Les fédérations de hand-ball, de tennis de table, de cyclisme, de Canoë-kayak et de course d'orientation disposant de tailles et de caractéristiques différentes pour illustrer la diversité des fédérations.

${ }^{8}$ Le rôle politique signifie une mission liée au portage du projet associatif (définition des valeurs, grands objectifs et orientations et contrôle des résultats) ; le rôle managérial concerne le management d'activités ou de projets (direction de projets ou/et d'équipe) et le rôle opérationnel (des tâches d'exécution : transport, secrétariat, accueil...).
} 


\section{Encadré 1 - Gouvernance des fédérations sportives (principaux éléments étudiés)}

Enquête quantitative (2005)

(1999 et 2003) 60 réponses/95 (via CNOSF)

Profil socioprofessionnel des principaux élus

Ancienneté et position antérieure dans le système fédéral Temps de travail hebdomadaire consacré

Rémunération/Indemnisation des élus

Nombre de membres bureau et comité directeur

Nombre de femmes au bureau et comité directeur

Nombre de réunions annuelles du bureau

et du comité directeur fédéral

Nombre de commissions de travail

Nombre de salariés et rôle des principaux cadres

Qualité perçue des relations de travail bénévoles et salariés

Formalisation du projet associatif

Nombre de partenariats avec des ministères

\section{Enquêtes qualitatives}

11 cas

Rôles (politique, managérial et opérationnel)

Études des relations entre élus, entre

élus

et cadres salariés

Travail du bureau et du comité

directeur

Dysfonctionneme nts perçus en termes de gouvernance (et avis sur l'évolution des systèmes électifs)

Au total près de 90 entretiens ${ }^{9}$ entre 1995 et 2009 ont été menés dont une cinquantaine avec des cadres et directeurs salariés, une trentaine de dirigeants bénévoles et une dizaine avec des experts du secteur (cadres du ministère des sports et du CNOSF et partenaires privés travaillant avec les fédérations qui fournissent une vision experte plus indépendante et libre du fonctionnement des fédérations). L'analyse demandée aux acteurs des FSF à partir de leur mémoire et de leur interprétation de la réalité sur une longue période est nécessairement sélective. Cette analyse pourra être rationalisée a posteriori et témoigner d'une autojustification constitutive d'un biais de recherche très important. C'est pourquoi, il est nécessaire de le réduire en triangulant les données et en recherchant la confirmation d'une information auprès de plusieurs personnes (Huberman et Miles, 1990).

${ }^{9}$ Ces entretiens ont porté essentiellement sur l'exercice du pouvoir dans la fédération, le rôle et missions des acteurs, du bureau et du CA. Nous avons cherché à comprendre comment était vécue la tutelle du ministère des sports et son influence sur la gouvernance. 
La période étudiée s'étend principalement du milieu des années 1990 à aujourd'hui permet de mesurer les changements et évolutions. Ce travail de recherche a été mené dans un contexte changeant (pressions commerciales croissante, nombreuses évolutions législatives...) et particulièrement sensible en termes de gouvernance. En effet, les dirigeants des fédérations sportives en France comme à l'étranger (notamment les présidents des fédérations internationales de football, de volley-ball cf. Arcioni, 2007) ont parfois été mis en cause pour l'efficacité de leur gestion et plus rarement pour des fautes de gestion personnelle.

Ainsi, le Président du Comité national olympique et sportif (CNOSF) entre 1992 et 2008, le Président de la Fédération française de tennis (de 1992 à 2008) ont fait récemment l'objet d'une condamnation pour abus de confiance et prise illégale d'intérêts ; une peine de six mois d'emprisonnement avec sursis et 10000 euros d'amende a été infligée aux anciens président et trésorier de la fédération française de football (1998-2006) pour avoir commis sciemment de faux bilans comptables de la fédération. La gouvernance des plus grandes fédérations sportives a donc été contestée alors que de nombreuses réformes dans la gouvernance des fédérations sportives ont eu lieu au cours des quinze dernières années afin d'améliorer la gestion de ces organisations et de s'assurer de la qualité des engagements de leurs dirigeants.

\section{État des lieux de la gouvernance des fédérations sportives françaises}

Les pratiques de gouvernance des FSF sont très encadrées d'un point de vue juridique notamment par le biais de statuts types liés à la délégation de service public attribuée aux FSF. Nous faisons ici référence à l'importance de l'influence de la gouvernance politique sur la gouvernance organisationnelle. Au cours de ces quinze dernières années, un nombre important de textes et de réformes ont proposé d'initier de nouvelles pratiques et de nouveaux principes de gouvernance (1). L'analyse des pratiques actuelles montre, malgré l'évolution de l'arsenal législatif, que des difficultés sont toujours présentes (2) et risquent d'affaiblir la qualité du travail des instances de gouvernance et au-delà le potentiel de performance de ces organisations.

\section{Poids de la tutelle publique et tâtonnements législatifs}

Depuis plus qu'une cinquantaine d'années, l'État ${ }^{10}$, par le biais de la voie législative, a tenté de fixer plusieurs règles de gouvernance. Dès 1945, les FSF devaient adopter des statuts-types obligatoires, réfléchir aux conditions de renouvellement des mandats de présidents et voyaient l'âge de leurs administrateurs limité. Les statuts-types auxquels les groupements sportifs étaient tenus de se conformer de 1945 à 1975 interdisaient la réélection des dirigeants fédéraux. Les statuts-types prévus par le décret du 3 juin 1976 levèrent cette interdiction, mais instituèrent un plafonnement de l'âge des dirigeants :

\footnotetext{
${ }^{10}$ Le Secrétariat d'Etat des Sports a fait l'objet de différentes appellations ; en 2002, Ministère de la jeunesse, des sports et la vie associative (MJSVA), il redevient depuis 2007 secrétariat d'Etat aux sports dépendant désormais pour la première fois du Ministère de la Santé.
} 


\begin{abstract}
- $20 \%$ au moins des membres du comité directeur devaient à la date de leur entrée en fonction soit avoir moins de 35 ans, soit n'y avoir été élus précédemment ;

- l'âge du président à son entrée en fonction devait être fixé à 70 ans maximum. Ces mesures semblent, en pratique, n'avoir pas toujours été appliquées. En 1985, toute tentative d'intervenir sur l'âge des dirigeants fédéraux par le moyen des statuts-types fut abandonnée. Le décret du 13 février 1985 relatif aux statuts des fédérations sportives françaises ne visait plus à assurer un renouvellement des dirigeants, mais seulement à assurer la représentation de toutes les catégories de licenciés (femmes, jeunes, athlètes, médecins...) au sein des organes de décision.
\end{abstract}

En 1995, le décret de 1985 a été abrogé et les statuts-types des fédérations sportives ont été modifiés afin de leur laisser une marge de liberté plus importante dans l'organisation de leur gouvernance organisationnelle. Cette évolution permettait d'agréer des établissements commerciaux de façon à les rattacher au mouvement fédéral, de reconnaître des licenciés non adhérents à un groupement sportif affilié à la fédération et de créer des organismes nationaux pour la gestion de disciplines connexes. Mais une des principales évolutions est le fait que les fédérations disposaient désormais, dans le choix de leurs statutstypes, de deux options dites « $A$ » ou « $B$ » qui changent les conditions de répartition des pouvoirs entre les instances dirigeantes. L'option $A$ correspondait au système de gouvernance traditionnel où le comité directeur dispose des pouvoirs de décisions stratégiques que le bureau met en application. L'option $\mathrm{B}$ visait dans son esprit à conférer aux fédérations un nouveau type d'organisation avec un conseil fédéral et un bureau exécutif. L'analogie avec la société à directoire et à conseil de surveillance est manifeste bien que le législateur ait veillé à ce que les présidents ne soient pas trop impliqués personnellement dans les affaires commerciales connexes à celles de la fédération en énumérant un certain nombre d'incompatibilités entre le mandat de président et l'exercice de certaines professions afin d'éviter des conflits d'intérêts problématiques :

"Les fonctions de chef d'entreprise, de président du conseil d'administration, de président du conseil de surveillance, d'administrateur, de directeur général, de directeur général adjoint ayant exercé dans des sociétés, des entreprises ou des établissements dont l'activité consiste principalement dans l'exécution de travaux, la prestation de fournitures ou de services pour le compte ou sous le contrôle de la fédération, de ses organes internes ou des clubs qui lui sont affiliés, sont incompatibles avec le mandat de président de fédération ».

Dans l'option B, les membres du bureau exécutif étaient nommés sur proposition du président. L'exigence de bénévolat des membres du bureau exécutif explique que ni le directeur général de la fédération ni les directeurs salariés ou le Directeur technique national (DTN), cadre mis à disposition par l'Etat, ne pouvaient en faire partie avec une voie délibérative. Malgré un intérêt pour l'option $B$, seulement 5 fédérations délégataires sur plus de 70 l'ont finalement adoptée. 
Le décret $n^{\circ} 2002-760$ du 2 mai $2002^{11}$ abrogeant le décret de 1995 a supprimé la notion de statuts-types et propose de nouveaux statuts comportant désormais des dispositions obligatoires laissant une plus grande liberté d'organisation aux fédérations. Désormais, les établissements agréés peuvent participer, avec voix consultative, au comité directeur fédéral dont ils peuvent occuper au plus $20 \%$ des sièges (si pas de délivrance de licence $10 \%$ des sièges). Le décret du 7 janvier 2004 impose une commission électorale indépendante et la présence d'un pourcentage de femmes élues en fonction du nombre de licenciés. II a également reconnu la possibilité de rémunérer des dirigeants assurant une mise en conformité avec une loi de finances promulguée à la fin de l'année 2002.

En effet, l'article 6 de la loi de finances du 30 décembre 2002, modifiant l'article 261 du CGI, permet, sans remise en cause le critère de gestion désintéressée, sous condition ${ }^{12}$, la rémunération des dirigeants. Une dizaine de présidents de fédérations commencent à être rémunérés dans les limites de ce dispositif.

Ce système d'indemnisation est pratiqué en Espagne et en Italie ainsi que dans quelques fédérations internationales. La possibilité de rémunération ouverte n'a pour l'instant pas changé la « fonctionnarisation " déjà marquée des présidents de fédérations et une difficulté des "jeunes" présidents venant du secteur privé à s'imposer. Ainsi notre étude montre qu'un peu plus d'un tiers d'entre eux sont fonctionnaires ou assimilés. Certains sont mis à disposition pour exercer leur fonction bénévole et d'autres disposent d'un emploi du temps aménagé. Un autre tiers est retraité (parfois du secteur public) et un dernier tiers vient du secteur privé.

\section{Des pathologies bien présentes}

Malgré ces évolutions, l'analyse de la gouvernance des fédérations sportives témoigne d'un certain nombre de difficultés qui montrent que les conditions de pilotage de la performance ne sont pas toujours créées au sein des FSF. II n'est pas rare que la politique de gouvernance soit à l'origine des difficultés, notamment financières, rencontrées dans un certain nombre de fédérations sportives (haltérophilie, sport de glace, ski...) depuis ces dernières années. Nos analyses des fédérations ont montré essentiellement cinq pathologies étroitement imbriquées qui peuvent être à l'origine des déficits de performance :

\footnotetext{
${ }^{11}$ Les décrets $n^{\circ}$ 2002-648 du 29 avril 2002 et les décrets du 2 mai $n^{\circ}$ 2002-761 et 2002-760 abrogent le décret du 13 février 1985 (voir le commentaire de F. Alaphilippe, Agrément et délégation nouvelle manière : leur incidence sur l'organisation des fédérations sportives, Revue Juridique et Economique du Sport, 2002, 64, 31-48).

${ }^{12}$ Ce principe est impossible pour des ressources inférieures à 200000 euros. II est possible pour :

- une personne pour des ressources comprises entre 200000 et 500000 euros ;

- deux personnes pour des ressources comprises entre 500000 et 1 million d'euros ;

- trois personnes pour des ressources supérieures à 1 million d'euros.

Ce principe devra être adopté à la majorité des $2 / 3$ par l'assemblée générale. Le montant des rémunérations individuelles ne pourra excéder trois fois le plafond de la sécurité sociale 103860 euros annuellement. C'est à dire 8655 euros mensuels pour 2010.
} 
- une politique et des pratiques de gouvernance mal définies : des conseils d'administration peu actifs dans leur rôle central d'orientation, de leadership et de contrôle. Des bureaux où les membres sont seulement choisis parmi les membres du CA au risque d'un manque de professionnalisme (en termes de temps ${ }^{13}$ et de compétences). Le système électif actuel de démocratie indirecte se traduit toujours dans les faits par une surreprésentation dans ces instances de gouvernance des élus régionaux et/ou départementaux. Cette situation comporte le risque de favoriser les conflits d'intérêts ${ }^{14}$, un manque de compétences, d'indépendance au sein des CA et des bureaux d'autant plus que les rôles de ces administrateurs sont parfois non ou mal définis et que le cumul des fonctions est souvent important (membre du comité directeur fédéral, président de ligue voire parfois également de club ou/et arbitre).

- des professionnels prennent, officiellement ou officieusement, la direction politique de ces organisations ; cette situation s'explique soit par l'abandon du pouvoir par les élus ou/et par l'ancienneté, le charisme, les qualités et le profil du directeur (directeur général ou directeur technique national la plupart du temps).

- des présidents parfois omnipotents (désormais pour quelques-uns uns, rémunérés) se comportent en véritable président directeur général, parfois sans l'existence de réels contrepouvoirs (tels qu'un CA fort et indépendant ou un contrôle et une évaluation précise de la tutelle publique). La légitimité électorale confère souvent une autonomie quasi-totale au président fédéral ; le bureau est une équipe acquise à sa cause et le comité directeur joue souvent peu son rôle de contrôle. Le risque de déviance en pouvoir autocratique et/ou d'un pilotage clientéliste aux détriments des " propriétaires » (les clubs), voire au-delà du contribuable (financeur direct et indirect souvent non négligeable) est alors possible. Cette situation traduit des pratiques d'enracinement ${ }^{15}$ que l'on retrouve dans les entreprises et les organisations publiques (Paquerot, 1996). Ce risque est d'autant plus important que les dirigeants en place sont particulièrement difficiles à évincer du pouvoir. En effet, les scrutins de liste (plutôt qu'uninominal) et le système électif sont basés sur de grands électeurs (sur les structures régionales ou/et départementales) et très rarement sur les clubs (seulement 2 cas sur 100 fédérations : la FF de rugby et la FF d'équitation). Cela explique que le système électif soit

\footnotetext{
${ }^{13}$ Selon notre enquête menée auprès des fédérations en 2005 (60 réponses sur 94 fédérations), le temps moyen hebdomadaire (temps de " travail », déplacements etc...) consacré à la fédération serait le suivant : env. $30 \mathrm{H}$. pour le président fédéral ; 22 heures pour le secrétaire général et 11.5 pour le trésorier. Pour les présidents de fortes disparités sont à noter $(30 \%$ passent plus de $41 \mathrm{H}$. par semaine pour leur fédération ; $14 \%$ entre 31 et $40 ; 29 \%$ entre 21 et $30 ; 18 \%$ entre 11 et 20 et $9 \%$ entre 0 et $9 \mathrm{H}$. (Bayle et Bruzek, 2005).

${ }^{14}$ II y a là une ambiguïté évidente qu'ont soulignée quasiment tous les responsables de fédérations que nous avons rencontrés. Les dirigeants fédéraux, élus au CA, qui représentent leur ligue (généralement), ont tendance à défendre les intérêts de leur structure " d'origine ", parfois même contre l'intérêt fédéral. Plus globalement, la dissociation des intérêts particuliers de l'intérêt général au sein du système fédéral apparaît particulièrement difficile à réaliser. On retrouve là un problème identique au domaine politique pour les députés qui représentent leur circonscription électorale.

${ }^{5}$ L'enracinement, qui s'inscrit dans le cadre de la théorie de l'agence ainsi que celle des coûts de transaction, suppose que les acteurs développent des stratégies pour conserver leur place dans l'organisation et évincer d'éventuels concurrents.
} 
facilement contrôlable par les élus en place. La rémunération des présidents de fédération entraîne également de fait une professionnalisation de la fonction qui entraîne les mêmes conséquences qu'en politique. La fonction de Président devient un " métier " plus facilement exerçable par des fonctionnaires.

- Une absence de clarté dans la délégation managériale. La délégation managériale du «politique » (les élus du CA) vers le staff exécutif salarié (qui contribue parfois largement à la mise en œuvre et au contrôle des activités) est mal ou non définie. En conséquence, le pouvoir managérial et d'engagement des directeurs ou responsables permanents est souvent faible et partiel et les jeux politiques perturbent le processus de décision (nous retrouvons ici le « modèle de la poubelle » identifié par Cohen et al. dans les années 1970 au sujet des universités américaines).

- Un risque de rupture, de sclérose et de conflits entre le sommet (le siège fédéral) et la base (les clubs - mandants) pouvant entraîner un affaiblissement de la démocratie (désintérêt des clubs), une incapacité/manque de volonté à mettre en œuvre les projets et la stratégie pensés parfois uniquement au niveau national. Les structures régionales et surtout départementales, qui jouent un rôle de relais de la politique fédérale et de représentation des structures locales, sont souvent trop faiblement professionnalisées pour accompagner efficacement la gouvernance du réseau fédéral.

- Un rôle incitatif et de contrôle ambivalent du ministère de tutelle : Si le Secrétariat d'Etat aux Sport souhaite garder un contrôle politique sur le secteur, il doit laisser ces organisations dans une certaine dépendance vis-à-vis de son action (en jouant sur les variables législatives, financières, politique...) (Bayle \& Durand, 2004).

Les quatre premières pathologies relèvent plutôt de la gouvernance organisationnelle alors que la dernière tient à la gouvernance politique. II est à noter également une difficulté à rentrer pour les FSF dans une logique de gouvernance systémique et à gouverner les relations de réseau entre différentes parties prenantes qui en découlent.

Ces symptômes sont le plus souvent les révélateurs d'une crise latente du modèle de gouvernance traditionnel des grandes FSF et du système sportif français. C'est le projet même de ces organisations qui risque de se perdre dans la confusion des moyens et des finalités que les CA des FSF ne portent, ne défendent et/ou ne contrôlent plus. Si de nombreuses fédérations ont réussi à trouver des modèles de gouvernance et de management originaux autour de compromis (un leader ou une équipe charismatique de bénévoles et de professionnels s'investissant de manière parfois « héroïque » en termes de temps d'investissement pour la cause fédérale), ces modèles restent souvent fragiles car liés à la présence de quelques personnalités très investies. Le modèle " traditionnel " (fort investissement des élus et des bénévoles dans la gouvernance et le management) a certes encore parfois du sens dans des systèmes peu professionnalisés pour faire émerger une représentation légitime et administrer l'organisation. Mais lorsque la taille 
et le nombre de salariés ainsi que les enjeux médiatiques et économiques sont plus importants, les pathologies évoquées peuvent apparaître plus facilement.

Des axes de propositions pour refonder la politique de gouvernance des plus grandes FSF peuvent être trouvés dans les évolutions parfois radicales adoptées au sein des systèmes de gouvernance des entreprises françaises (notamment le rapport Viennot, $1999^{16}$ ) ou des associations et des fondations (IFA, 2009) mais surtout au sein des réformes anglosaxonnes concernant les organisations à but non lucratif (non profit organisations).

Des éléments de réponse dans le monde anglosaxon...
Pour faire face à des problèmes identiques à ceux des FSF, depuis le début des années 2000 , de nouvelles pratiques de gouvernance émergent dans les associations en général et dans les fédérations sportives et comités olympiques anglo-saxons sous l'influence de la doctrine « Carver 117 .

\section{La doctrine Carver}

Les principes généraux de cette doctrine sont les suivants:

- $\quad$ Privilégier des conseils d'administration (CA) de taille réduite (une petite dizaine de personnes en général) :

- $\quad$ Composition du CA par des élus majoritairement indépendants (qui n'ont pas d'intérêts financiers directs ou indirects dans la gestion de l'organisation) ;

- $\quad$ Création de postes de spécialistes au CA (experts-comptables et financiers, marketing, indépendants...);

- $\quad$ Supprimer la notion de bureau ;

- $\quad$ Suppression des postes de trésorier, de vice-président et de secrétaire général présents dans le modèle traditionnel; dans le nouveau modèle, il y a uniquement la fonction de président du CA ;

- $\quad$ Limitation de la durée des mandats des élus (en pratique, pas plus de 8 ans dans le CA pour un administrateur ${ }^{18}$ );

- Délégation managériale à un directeur général - DG - (chief executive officer) qui rend compte au CA des résultats obtenus et des conditions d'obtention des résultats ;

- Le CA contrôle le DG, mais se concentre sur des questions politiques et stratégiques comme le contrôle des résultats obtenus.

Ces nouveaux principes témoignent de trois axes forts dans les réformes de gouvernance :

\footnotetext{
${ }^{16}$ Le rapport Viennot (1999) a inspiré la loi « NRE » (« nouvelles régulations économiques ») de juillet 2001.

${ }^{17}$ Nom d'un universitaire américain ayant travaillé sur les principes et méthodes visant à améliorer la gouvernance des organisations à but non lucratif $(1997,2001)$.

${ }^{18}$ Aux Etats-Unis, un comité de nomination indépendant coopte ainsi un certain nombre de candidats sur les postes à pourvoir ; ils sont ensuite élus par l'assemblée générale des clubs.
} 
- Mieux clarifier les responsabilités de gouvernance et de management ;

- Favoriser l'évaluation, le contrôle et la transparence de la performance ;

- $\quad$ Assurer la diversité des représentants du CA et s'assurer de la présence d'un minimum de compétences pour améliorer l'efficacité du travail du CA.

Depuis la fin des années 1990, deux tiers des fédérations sportives notamment canadiennes ${ }^{19}$ et américaines mais aussi d'autres pays anglosaxons, Australie ${ }^{20}$ et Nouvelle-Zélande, ont adopté ces principes de gouvernance. La réforme du Comité International Olympique (« ClO 2000 ») s'en est également inspirée sans réellement l'appliquer (révision de la composition des membres, limitation de la durée du mandat du président à 8 ans +4 ans maximum, formalisation obligatoire d'un plan stratégique) à la différence du comité olympique américain (USOC) ${ }^{21}$.

\section{L'exemple d'application en Grande-Bretagne}

Par exemple, la Grande-Bretagne a fait une application adaptée de ces mesures. Les fédérations anglaises disposent d'un certain nombre de différences dans leurs structures : la taille du CA, la composition du CA en termes de personnes élues et de bénévoles nommés, le rôle du président en tant que leader et responsable du CA, le rôle des salariés au CA, la présence du président qui est élu ou nommé et qui peut être rémunéré, la présence de membres indépendants qui peuvent être rémunérés ou non.

Par exemple, une fédération anglaise dispose d'un CA qui comprend un certain nombre de bénévoles élus, d'autres nommés, un président bénévole qui a été recruté à l'extérieur du sport et un président qui est une " tête d'affiche " (figure head) et qui n'a pas de droit de vote. Les personnels salariés agissent en tant que secrétaire et trésorier du CA, d'autres membres clés du personnel salarié rejoignent le CA en qualité de conseils, mais aucun membre du personnel salarié n'a le droit de vote. Une autre fédération dispose d'un CA beaucoup plus petit qui comprend seulement des membres élus : le président agit en qualité de président du $\mathrm{CA}$ et le Directeur général est au CA. Ces deux exemples témoignent qu'il n'y a pas un modèle unique de gouvernance dans les fédérations sportives britanniques.

De cette diversité des structures de gouvernance, UK Sport (organisme en charge du sport anglais), suite au rapport Deloitte et Touche (2003), a mis en évidence un certain nombre de principes de gouvernance que toutes les fédérations sont censées réaliser. La gouvernance efficace prend en compte la gestion de la structure, l'adhésion aux meilleures pratiques, un plan stratégique à long terme, un rapport relatif aux

19 Voir J. Duncan, L'évolution de la gouvernance des conseils d'administration des organisations sportives canadiennes in La Gouvernance des organisations sportives, L'Harmattan, Dir. Bayle et Chantelat, 2007.

${ }^{20}$ Voir par exemple : Australian Sports commission (http://www.ausport.gov.au.)

${ }^{21}$ Voir l'entretien B. Gambardella dans la Lettre de la Revue Juridique et Economique du Sport, 6, 2005. 
orientations stratégiques et un rôle et des responsabilités claires attachées au Directeur général. Cependant, étant donné la diversité des modes de gouvernance des fédérations, le développement d'un cadre commun de gouvernance n'a pas été jugé approprié et, dans un premier temps, UK Sport a décidé de se focaliser prioritairement sur la structure du CA. Un certain nombre de principes ont été proposés qui doivent être mis en œuvre quelle que soit leur structure de gouvernance. II s'agit des principes suivants :

- La structure du CA devrait généralement être composée de 5 à 10 membres plus un Président. Le nombre de membres du CA devrait refléter la taille et le niveau d'activité de l'organisation ;

- $\quad$ En complément de ces 5 à 10 personnes, le CA devrait rechercher à nommer 3 membres extérieurs qui disposent de compétences professionnelles essentielles pour le CA;

- Les membres du CA ne doivent pas seulement représenter les membres, mais ils doivent avoir les compétences nécessaires pour mener à bien leur rôle dans la gouvernance. Ces compétences peuvent être d'ordre financier, légal, commercial, de la communication ou des nouvelles technologies ou autres compétences nécessaires pour la fédération ou son sport ;

- Les membres du CA doivent autant que possible représenter la diversité de la population et la diversité géographique et technique de leur sport ;

- Les membres du CA doivent être nommés pour une durée spécifique et sans renouvellement automatique, pour encourager le renouvellement du CA tout en conservant la mémoire organisationnelle. En plus, les nominations au CA doivent être fixées de manière à ce que les nouvelles nominations ne coïncident pas ;

- $\quad$ Les membres du CA doivent recevoir une formation minimale d'un standard reconnu et par une autorité accréditée dans les 6 mois suivant leur nomination ;

- $\quad$ II doit y avoir un plan de succession des membres du CA ;

- II est essentiel que les membres du CA soient présents aux réunions et fournissent une assistance dans le processus de décision. Les membres du CA qui manquent plus de trois CA consécutifs doivent être interrogés sur leur place dans le CA, à moins qu'ils ne justifient de circonstances exceptionnelles ;

- $\quad$ Des sous-comités doivent être créés pour répondre aux besoins et exigences du travail du CA ; ils doivent soumettre leurs comptes- rendus au CA.

Cependant, il est important de noter que ces principes doivent être appliqués en tenant compte de la taille et du rôle de la fédération. Ils n'ont pas vocation à créer une structure standard de gouvernance de chaque fédération, mais se présentent plutôt comme un guide pour améliorer la gouvernance dont la fédération a besoin.

Au regard des pathologies mises en évidence et des solutions présentées à l'étranger, la dernière partie de l'article vise à dessiner les évolutions souhaitables et possibles de la gouvernance des FSF. 
Quelles évolutions de la gouvernance des FSF?
Les principes de la doctrine Carver et leur adaptation dans le cas britannique ne sauraient s'appliquer à toutes les FSF car bon nombre d'entre elles ont parfois du mal à fidéliser et à recruter des dirigeants bénévoles. Mais certains de ces principes peuvent être adaptés pour des FSF très professionnalisées pour lesquelles les enjeux médiatiques et économiques sont devenus substantiels (fédérations françaises de football, de tennis...). Cependant, la tutelle de l'Etat et la délégation de mission de service public leur imposent encore un cadre rigide et unique de fonctionnement et bien des dirigeants interrogés sur la doctrine Carver la trouvent trop « révolutionnaire " voire " contraire aux principes de la loi du $1^{\text {er }}$ juillet $1901 »^{22}$.

L'ancien Secrétaire d'Etat aux Sports, B. Laporte, avait annoncé, en 2008, juste avant les élections fédérales qui suivent les Jeux Olympiques, son intention de réformer à nouveau la gouvernance des FSF. La réforme envisagée était de limiter le nombre de mandats du président fédéral à deux mandats successifs de quatre ans (à l'instar de ce que commande la constitution pour le Président de la République). Ce projet législatif témoigne d'une manière symptomatique de poser les projets de réforme sur un sujet sensible : d'une part, proposition d'une solution partielle sans analyse précise de l'ensemble des problèmes et d'autre part, absence de concertation avec le mouvement sportif à un moment de tension lié aux élections (proposition vécue comme une immixtion politique intolérable).

Le mouvement sportif et les présidents de fédérations disposent, en effet, d'une position très paradoxale en acceptant les moyens publics substantiels (subventions et fonctionnaires mis à disposition) sans en accepter toutes les contraintes (contrôle et systèmes d'influences).

Il faut dire que la capacité des FSF et plus largement du secteur associatif à s'auto-réformer peut sérieusement être mise en doute. Les possibilités de réformes en matière de gouvernance apparaissent plus lors de crise (médiatique) majeure où l'opinion publique est directement interpellée (malversations, dysfonctionnements démocratiques majeurs, détournements des finalités, performances faibles...). Dans d'autres secteurs associatifs, la création du comité de la charte et l'acceptation de son pouvoir de régulation s'est par exemple réalisée dans un tel contexte de crise (image très écornée du monde associatif suite à l'affaire de l'Arc) ; il en est de même quant à la gouvernance du $\mathrm{ClO}$ très fortement mise à mal à la fin des années 1990 et qui a du donner des gages de changements (création d'une commission éthique et participation à la création d'une agence de régulation des problèmes de dopage : l'Agence mondiale antidopage - voir Chappelet, 2002). II semble que des agences ou acteurs indépendants (type coordination inter-associative) soient le gage d'une régulation plus efficace.

Des organismes experts et des travaux universitaires peuvent également jouer un rôle pour promouvoir des bonnes pratiques bien que la diffusion de ces travaux auprès des dirigeants associatifs soit parfois compliquée. Le travail de l'IFA vise ainsi à améliorer les pratiques de gouvernance des

\footnotetext{
${ }^{22}$ Source : entretiens avec des deux présidents.
} 
associations et des fondations notamment en assurant mieux la qualité des engagements requis des administrateurs. Cet ouvrage sous la forme de guide pratique présente des expériences en matière de recrutement des administrateurs et quant à la composition du CA (limite d'âge, durée des mandats limitée à 2 fois 3 ans, limitation du cumul des mandats).

Pour favoriser le fonctionnement du CA et un meilleur équilibre des pouvoirs, la question du nombre et de la durée des réunions ainsi que la possibilité de recourir à des comités de conseil, de missionner un administrateur ou de mandater un expert indépendant est proposée. Les auteurs notent cependant que « la mise en place de comités de conseil ainsi que le recours à un administrateur missionné ou à un expert indépendant s'apprécient en fonction de la taille de l'association, de sa culture et de son environnement ». Les auteurs du guide insistent sur la nécessaire formalisation des délégations de pouvoirs. Ils proposent également la formalisation d'une charte des administrateurs qui a vocation à mieux définir les droits et devoirs des administrateurs ainsi qu'à exposer les principes organisationnels qui en résultent $A u$ cours de notre étude, nous n'avons trouvé ces pratiques qu'au sein d'une seule fédération sportive (la fédération française de randonnée pédestre) sur les onze cas étudiés.

L'ouvrage de I'IFA promeut également des pratiques qui peuvent apparaître encore nouvelles pour les FSF : nécessaire formalisation du projet associatif (seules 1/3 le font Bayle et Bruzek, 2005) et définition des structures et personnes qui conçoivent, mettent en œuvre et contrôle la réalisation du projet associatif.

Quant à l'identification et le contrôle des risques dans l'organisation et à l'analyse de la valeur et de l'utilité sociale, elles paraissent encore très (trop ?) pionnières et innovantes dans l'univers des FSF.

Si la question du contenu de la réforme de la gouvernance des FSF est fondamentale, celle des modalités l'est donc tout autant. Le sujet est sensible car il remet en cause les dirigeants actuels dans leur fonctionnement et dans leur avenir politique associatif.

Les grandes organisations associatives en général et sportives en particulier ne peuvent donc échapper à des réflexions et des réformes qui se posent ou se sont posées, en France mais aussi et surtout dans les pays anglo-saxons, quant à la gouvernance des (grandes) entreprises mais aussi des associations.

Le travail d'étude réalisé montre sur les plans théorique et empirique l'intérêt d'intégrer trois niveaux dans l'analyse de la gouvernance des FSF : organisationnelle, politique et systémique. L'article présenté s'est essentiellement intéressé à la gouvernance organisationnelle et aux effets de la gouvernance politique mais aussi systémique sur la gouvernance organisationnelle des FSF. La question de la gouvernance systémique a certes été peu abordée dans la mesure où le ministère de tutelle est la partie prenante encore dominante et souvent prépondérante pour les FSF, baignées dans une culture et tradition de service public. 
Toutefois, les revendications de nouveaux stakeholders (sportifs professionnels, nouveaux médias, sociétés de paris sportifs, sponsors, opinion publique...) tout comme la rationalisation de l'action de l'Etat dans le cadre de la LOLF (Nikonoff et Bayle, 2007) expliquent l'exigence d'une capacité à fédérer et à concilier des intérêts et attentes de parties prenantes parfois contradictoires (entraînant des conflits potentiels de valeurs et des rationalités associées : marchand/non marchand, public/privé, lucratif/non lucratif, professionnalisme/militantisme, intérêt collectif/intérêts particuliers...). Cette situation explique d'autant plus les besoins d'une gouvernance organisationnelle professionnalisée et apte à répondre à ces défis.

Cette professionnalisation de la gouvernance se traduit par l'émergence de nouvelles questions essentielles quant à la " qualité " (accès, compétences et valeurs) des dirigeants, la séparation des pouvoirs entre le politique et le management, le contrôle des dirigeants par les " propriétaires ", la composition et le fonctionnement des conseils d'administration (CA), la relation entre les administrateurs (élus) et les managers, la capacité et la volonté de piloter la performance de ces organisations. Dans ce contexte et face aux exigences d'adaptations aux changements, la gouvernance est incontestablement (re)devenue une dimension et un facteur clé pour le pilotage de la performance de ces organisations. Cette forme de rationalité managériale reste parfois encore très étouffée et secondaire au profit d'une régulation politique qui s'opère souvent au regard des intérêts des acteurs (le Président et les principaux dirigeants). C'est pourquoi, les propositions présentées ne doivent pas entraîner une vision homogène et uniforme des réformes à mener. II s'agit plutôt d'ouvrir des questionnements et à un champ de possibilités qui doivent, avant d'être implantées, tenir compte d'un certain nombre de facteurs et d'éléments contextuels : la nature du projet, la taille, le cycle de vie, le niveau de professionnalisation, l'intensité conflictuelle et les jeux de pouvoir présents au sein de la FSF. Ces éléments donnent une idée des capacités, volontés et exigences de reformes en matière de gouvernance.

La dernière question a trait aux modalités du changement et aux capacités de réformes. L'ensemble des changements proposés dans les modèles anglo-saxons peuvent être certes suscités ou imposés par l'Etat que ce soit par la voie législative ou conventionnelle, mais les mécanismes de contrôle du système de gouvernance les plus efficaces semblent être ceux que les membres de la fédération auront su s'imposer à eux-mêmes et non ceux que le ministère de tutelle tentera de leur imposer. L'expérience montre que cette seconde éventualité est souvent mal vécue par le monde associatif. 


\section{BIBLIOGRAPHIE}

Amis, J., Slack, T., \& Hinings, C. R. 2004. Strategic Change and the Role of Interests, Power and Organizational Capacity, Journal of Sport Management, 18, p. 158-198.

Arcioni S. (2007), " La gouvernance des fédérations sportives internationales : proposition d'un cadre d'analyse ", Gouvernance des organisations sportives sous la direction d'E. Bayle et P. Chantelat, Paris : L'Harmattan, p. 53-67.

Adams, C.T. \& Perlmutter, F.D. (1995), "Leadership in hard times: Are non profits well served ?", Nonprofit and voluntary Sector Quarterly, n ${ }^{\circ}$ 24, p. 253-262.

Alaphilippe, F. (2002). « Agrément et délégation nouvelle manière : leur incidence sur l'organisation des fédérations sportives ", Revue Juridique et Economique du Sport, Eds Dalloz, n64, p. 31-48.

Bayle, E. (1999), Management et performance des organisations à but non lucratif : le cas des fédérations sportives nationales, thèse de doctorat de sciences de gestion, Université de Limoges.

Bayle E. et Camy J. (2003), Le dirigeant fédéral : aujourd'hui et demain, Rapport pour l'Académie nationale olympique française (ANOF) du Comité national olympique et sportif français (publication sur le site internet du CNOSF : http://www.comite-olympique.asso.fr).

Bayle et Durand (2004), " Vingt ans de relations entre le mouvement sportif et l'Etat : d'une collaboration ambivalente vers une régulation managériale ? ", Revue Politiques et management public, IMP, p. 125 à 144.

Bayle E. et Bruzek M, (2005), Le Management associatif - 4 défis, 15 enjeux et 65 actions pour le mouvement sportif, Eds. Comité National Olympique et Sportif Français

P. Chantelat, coll. Espaces et temps du sport L'harmattan, p.113-127

Carver, J. (1997), Boards That Make A Difference: a new design for leadership in non profit and public organizations, San Francisco, Oxford, Jossey Bass.

Carver, J., (2001), "Carver's Policy Governance Model in Nonprofit Organizations", The Canadian journal Gouvernance - Revue internationale, Vol. 2. nos. 1, p. 30-48 www.carvergovernance.com

Chait R.P., Ryan, W., \& Taylor, B.E. (2004). Governance as leadership: Reframing the work of nonprofit boards. Wiley \& Sons.

Chappelet, J.-L., 2002, « L'agence mondiale antidopage : un nouveau régulateur des relations internationales sportives ", Relations internationales, $\mathrm{n}^{\circ} 111$, p. 381-401.

Charreaux, G. (2003), "Le gouvernement des entreprises ", in J. Allouche (ed.), Encyclopédie des ressources humaines, Economica, Paris.

Charreaux, G. (dir.) (1997), Le gouvernement d'entreprises, Théories et faits, Economica, Paris.

Cohen M.D., March J.G., Olsen J.P. (1972), "A garbage can model of organizational choice", Administrative Science Quarterly, 17 (1), p. 125.

Courtois, G. (1997), « La théorie de l'agence appliquée aux associations ", Revue des études coopératives mutualistes et associatives, 40, p. $39-42$ 
Deloitte \& Touche "Investing in change » - high level review of the modernisation programme for governing bodies of sport, july 2003, report for UK sport (non published).

Demoustier D. (2001), L'économie sociale et solidaire, Editions La Découverte et Syros, Paris.

Duncan (2007), " L'évolution de la gouvernance des conseils d'administration des organisations sportives canadiennes " in La Gouvernance des organisations sportives Dir. Bayle et Chantelat, Eds L'Harmattan, p. 145-158.

Ernst et Young (2006), Quelle approche de la gouvernance dans l'univers des Organismes Sans But Lucratif ? (étude non publiée)

Fama, E., \& Jensen, M. (1983), " Agency problems and residual claims », Journal of Law and Economics, 26, p. 327-349.

Finet, A. (Dir.) (2005), Gouvernement des entreprises, De Boeck, Bruxelles.

Institut Français des Administrateurs (2009), Gouvernance des associations et fondations, Editions d'organisation.

Gomez, P.Y. (1996). Le gouvernement de l'entreprise, modèles économiques de l'entreprise et pratiques de gestion, Interéditions, Paris.

Gomez P.Y. (2001), La république des actionnaires, le gouvernement d'entreprise entre démocratie et démagogie, La Découverte \& Syros, Paris.

Green, J.C. \& Griesinger, D. (1996), "Board performance and organizational effectiveness in non profit social service organizations", Nonprofit Management and Leadership, 6, 381-402.

Henry, I.P. (2005), Governance in sport : a political perscpective, Institute of Sport and Leisure Policy, Loughborough University.

Herman, R.D. \& Heimovics, R.D. (1991), Executive leadership in Nonprofit organizations: New strategies for shaping executiveboard dynamics, San Francisco, Jossey-Bass.

Honta M. (2007), Sport et action publique : une analyse des modes de gouvernements territorial, HDR Université de Bordeaux 2.

Hoye, R. \& Cuskelly G. (2006), Sport governance, ButterworthHeinemann.

Huberman A.M. et Miles B. (1991), Analyse des données qualitatives, Recueil de nouvelles méthodes, De Boeck Université.

Kaltenbach P. (1996), Associations lucratives sans but, Denoël.

Kikulis, L. M. (2000), "Continuity and Change in Governance and Decision Making in National Sport Organizations:Institutional Explanations», Journal of Sport Management, 14, p. 293-320.

Kikulis, L. M., Slack, T., \& Hinings, C. R., (1992), «Institutionally specific design archetypes: A framework for understanding change in national sport organizations». International Review for the Sociology of Sport, 27, p. 343-370.

Kikulis, L. M., Slack, T., \& Hinings, C. R., (1995), « Sector-specific patterns or organizational design change». Journal of Management Studies, 32(1), p. 1-34.

Lipietz A. (2001), Pour le tiers secteur. L'économie sociale et solidaire : pourquoi ? comment? La Documentation française 
Mayaux F. (1996), Noyau stratégique des associations : quel partage des pouvoirs entre dirigeants bénévoles et dirigeants salariés?, Thèse de doctorat de sciences de gestion, Université Lyon III.

Mayaux, F. (1999), « Typologie des conseils d'administration d'associations ", Revue Internationale de l'Economie Sociale, 272, p. 45-57.

Nikonoff L. et Bayle E. (2007), «Pilotage de la performance publique : les premiers effets de la LOLF au sein du Ministère de jeunesse, des sports et de la vie associative ", Revue européenne de management du sport, n¹9, Eds. PUS.

Papadimitriou, D. (1999), « Voluntary boards of directors in Greek sport governing bodies», European Journal for Sport Management, Special issue, $6,78-103$

Paquerot M. (1996), " L'enracinement des dirigeants et ses effets ", Revue Française de

Gestion, nov/dec, n¹11, p.212-225.

Perez, R. (2003), Le gouvernement des entreprises, La découverte, coll. Repères, Paris.

Pesqueux, Y. (2007), Gouvernance et privatisation, PUF, Paris.

Queinnec, E. et Igallens, J. (Dir.), (2004), Les organisations non gouvernementales et le management, Vuibert.

\section{SITOGRAPHIES}

http://www.ccomptes/publications/rapports/rp2003/chapitreXI.pdf

http://www.hlst.Itsn.ac.uk/resources/governance.html

http://www.carvergovernance.com/model.htm

Australian Sports commission at http://www.ausport.gov.au

Football governance research centre in the UK at http://www.footballresearch.bbk.ac.uk/index.htm

Sport and recreation New Zealand at http://www.sparc.org.nz 